\title{
A plea for biological descriptions: the case of reproduction biology
}

\author{
Peter Nick ${ }^{1}$
}

Received: 1 October 2019 / Accepted: 1 October 2019 / Published online: 15 October 2019

(C) Springer-Verlag GmbH Austria, part of Springer Nature 2019

Diversity is a central feature that delineates biology from other natural sciences, such as physics. Whether a falling body is red or blue or shows some intricate stripe pattern on its surface is not relevant - this body is moving according to the laws of gravitational attraction. For a biologist, in contrast, the discriminative features of an object are of utmost relevance. This holds true, even though striking commonalities (culminating in the cell theory, genetics, or the rise of molecular biology) between life forms could be uncovered and have shaped the life sciences ever since. The diversity of life is tightly linked with the concept of 'species'. One would expect, therefore, that such a central concept as a biological 'species' should be unified. This is not the case - in fact, different species concepts coexist and somehow refuse to merge into one general definition, what a 'species' is supposed to be (for insightful review, see De Queiroz 2007). The diversity of species concepts is not owed to historical constraints, such as the negation of species transformation as it had been shaping the Linnean species concept (cf Ramsbottom 1938). The diversity of species concepts is rather due to the fact that biological species represent emergent properties of reproduction, i.e. of gene flow. Since reproduction is the core mechanism of any evolution, it represents the immediate lever of selection and, as a consequence, groups among the biological phenomena, where we face the most pronounced variation. Four contributions to the current issue illustrate this point for both, plant and animal reproduction, but pursue a different purpose.

Plant sexuality deviates in several aspects from the common paradigm of two genders that form meiotic germ cells that fuse and give rise to a new organism. Instead, most flowering plants are hermaphrodites, and in all land plants,

Handling Editor: Peter Nick

Peter Nick

peter.nick@kit.edu

1 Botanical Institute, Karlsruher Institut für Technologie, Karlsruhe, Germany the meiotic germ cells (called spores to delineate them from sperm and eggs) do not fuse immediately, but undergo mitotic divisions giving rise to a haploid gametophyte, who will then produce sexual organs, another round of (mitotically generated) germ cells that will fuse to initiate the new diploid organism. This so called alternation of generation contributing to the complexity of plant sexuality and making the life of botanists even more difficult is often accompanied by asexual or pseudosexual forms of propagation, where somatic cells develop into an embryo in a bypass around meiosis and fertilisation. These variations of the theme are of high practical relevance, because they have a strong impact upon breeding as illustrated by two of the contributions highlighted here: The work by Guo et al. (2019) in the current issue deals with jujube, a traditional fruit crop in China with high potential as functional food. The finding of a male-sterile cultivar called 'Dongzao' is of high practical relevance, because breeding in hermaphrodites means that one has to prevent self-pollination by emasculation, a tedious and error-prone task. Male-sterile genotypes are, therefore, a great tool for breeders but are problematic for subsequent application, because such, functionally female, plants will bear fruit only if a male plant is close by. To understand the mechanism behind the male sterility of 'Dongzao', authors conducted a comparative study of sporogenesis and gametophyte development, where the male-sterile cultivar was compared with the fertile cultivar 'Mayazao'. The authors report numerous new details of this process. Surprisingly, they find that the pollen grains (the male spores) develop and mature completely normally, which means that the reason for the male sterility must be searched for either in the release of the spores (so called anther dehiscence) or in the development of the resulting macrogametophyte (the pollen tube). Also, the work by Sutthinon et al. (2019) in the current issue is motivated by the need to understand male sterility, this case for mangosteen. This important tropical crop is endowed with a quite mysterious sexuality. All trees are female, and male trees are not known, a kind of plant version of the amazons. While the amazons, according to Strabo, once a year met and mated with their neighbouring tribe, the male Gargarians, 
the mangosteen uses apomixis, i.e. a pseudosexual form of propagation, where the embryo derives from somatic cells of the mother (Richards 1990). The flower starts from a (morphologically) hermaphroditic state but is functionally female by pollen abortion. Similar to the jujube study, the authors use a comparative approach, this time choosing G. celebes, a closely related and fertile species (and supposedly one of the wild parents of domesticated mangosteen) to understand the mechanism behind male sterility. Again, they report numerous, hitherto unknown details of microsporogenesis and can pinpoint, by numerous cytological details, a precocious cell death of the tapetum (the nutritive tissue for the pollen) as cause for male sterility. Thus, it is not the acquisition of a new gene which is responsible for the loss of male fertility, but a shift in temporal regulation of developmental processes that occurred during the hybridisation between the wild ancestors of mangosteens. As a recent review on male sterility puts it, 'it is a matter of timing' (Carrizo Garcia et al. 2016). Translated to the molecular level, temporal expression patterns of regulatory genes decide about sterility versus fertility. These depend on the respective promoters, which means that it should be possible to restore fertility and, thus, the possibility to improve mangosteen by breeding.

Animal taxonomy often relies on traits related to sexuality, because these traits often provide propagation barriers that decide the gene flow and thus speciation. Two contributions deal with the cellular details of spermatogenesis in the context of systematics and taxonomy. The work by Ben Ahmed et al. (2019) in the current issue follows spermatogenesis in the amphibian leech, Batracobdella algira. The development in the testis is asynchronous in this species, such that all stages can be studied side by side. They uncover dynamic remodelling of the microtubules during spermatogenesis and describe the ultrastructural details of the spermium. A comparison with other hirudinids shows a general overlap of the pattern, but diagnostic details in organelle length and shape not only are suited as taxonomic features, but also raise interesting functional questions related to the speciation of the leeches. The work by Miao et al. (2019) follows a similar approach, but for the hangingfly Terrobittacus implicatus. The hangingflies represent an extensive family, but due to numerous specialisations, their phylogenetic relationships are discussed controversially. To shed light into this debate, the authors dissect spermiogenesis and describe numerous cytological details. Again, specific changes of the mitochondria are uncovered and taxonomically relevant accessory bodies and mitochondrial structures in the acrosome can be described. Similar to the work on leech spermatogenesis, the authors compare the patterns to other species and discuss phylogenetic implications and discuss the value of some of these traits, for instance the number of so called accessory bodies, or the mitochondrial derivatives to resolve phylogenetic relationships.

All four contributions show impressively that details matter, when we want to study reproduction, and this, somehow, appears to be antagonistic to the otherwise strong tendency towards unification governing current biology. The appropriate approach to understand the diversity of reproduction is to study the details - thorough description has highest priority, therefore, and all four contributions live up to this task. The motivation behind these descriptions can be different. For instance, the two contributions to plant sexuality were driven by the intention to obtain a deeper insight into the causes for male sterility as prerequisite for future breeding endeavours. The two contributions on spermatogenesis use description to address evolutionary relationships. In both cases, description is not a value per se, but it always serves a purpose.

\section{Compliance with ethical standards}

Conflict of interest The author declares that there is no conflict of interest.

\section{References}

Ben Ahmed R, Malota K, Jarosz N, Świątek P (2019) Microscopic analysis of spermatogenesis and mature spermatozoa in the amphibian leech Batracobdella algira (Annelida, Clitellata, Hirudinida). Protoplasma, current issue

Carrizo Garcia C, Nepi M, Pactini E (2016) It is a matter of timing: asynchrony during pollen development and its consequences on pollen performance in angiosperms-a review. Protoplasma 254:5773

De Queiroz K (2007) Species concepts and species delimitation. Syst Biol $56: 879-886$

Guo Y, Li X, Huang FY, Pang XM, Li YY (2019) Megasporogenesis, microsporogenesis, and female and male gametophyte development in Ziziphus jujuba Mill. Protoplasma, current issue

Miao Y, Liu BP, Hua BZ (2019) Spermiogenesis of the hangingfly Terrobittacus implicatus (Huang \& Hua) (Mecoptera: Bittacidae). Protoplasma, current issue.

Ramsbottom J (1938) Linnaeus and the species concept. Proc Linnean Soc London 150:192-220

Richards AJ (1990) Studies in Garcinia, dioecious tropical forest trees: the origin of the mangosteen (G. mangostana L). Bot J Linn Soc 103:301-308

Sutthinon P, Samuels L, Meesawat U (2019) Pollen development in male sterile mangosteen (Garcinia mangostana L.) and male fertile seashore mangosteen (Garcinia celebica L.). Protoplasma, current issue

Publisher's note Springer Nature remains neutral with regard to jurisdictional claims in published maps and institutional affiliations. 